\title{
INTEGRACIÓN E INFRAESTRUCTURA EN AMÉRICA DEL SUR. APORTES PARA EL ESTUDIO DEL ROL ESTRATÉGICO DE LA HIDROVÍA PARAGUAY- PARANÁ EN LA INTEGRACIÓN REGIONAL
}

\section{INTEGRATION AND INFRASTRUCTURE IN SOUTH AMERICA. A CONTRIBUTION TO THE STUDY OF THE STRATEGIC ROLE OF THE PARAGUAY-PARANÁ WATERWAY IN REGIONAL INTEGRATION}

Álvaro Álvarez

\section{RESUMEN}

En las últimas décadas un aspecto central en la discusión sobre la integración regional es el lugar preponderante otorgado a la integración en infraestructura, en compatibilidad con la implementación en la región de modelos de inserción en la economía internacional reprimarizados (Bolinaga \& Slipak, 2015) que necesitan la reconversión de los corredores existentes en relación a las nuevas dinámicas de circulación.

En este contexto y, siendo la multimodalidad la característica más significativa del transporte internacional, las vías fluviales recobran importancia central como una forma de disminuir costos de circulación y como un factor clave en la integración de los territorios.

Este trabajo centrará su análisis en el rol geopolítico y comercialmente estratégico de la Hidrovía Paraguay-Paraná y en las obras de infraestructura planificadas y en desarrollo sobre los ríos Paraguay y Paraná en las últimas décadas. Para ello se utilizará una metodología de investigación multiescalar (García, 2006) y de triangulación (Bericat, 1998) combinando el análisis de fuentes y documentos oficiales de instituciones como CEPAL, CAF, BID, IIRSACOSIPLAN con entrevistas a informantes claves.

La discusión nodal emergente es qué infraestructura se promueve y financia en la región y para qué tipo de integración, en un contexto de debate paradigmático.

\section{PALABRAS CLAVE}

Integración, Infraestructura, Circulación, Hidrovía, Reprimarización.

\section{ABSTRACT}

Since the last decades, a key aspect regarding regional integration has been the role of infrastructure integration in consonance with regional economic models that achieve insertion in global economy through reprimarization (Bolinaga \& Slipak, 2015) and that need the conversion of current corridors to adjust to new transportation dynamics.

In this context, and multimodality being the most significant feature of international transportation, waterways become central as an alternative that decreases transportation costs and as a key factor in territory integration.

This work focuses on the geopolitically and commercially strategic role of the Paraguay-Paraná Waterway and on the infrastructure projects planned and under development on Paraguay and Paraná rivers since the last decades. Multi-scalar methodology (García, 2006) and triangulation (Bericat, 1998) are used; source analysis and official documents from institutions such as CEPAL, CAF, IIRSA-COSIPLAN are used along interviews with key informants.

The issue here discussed, which is becoming increasingly important in a context of paradigmatic debate, is which types of infrastructure are being fostered and financed in the region, and for which kind of integration. 

DOI: https://doi.org/10.24215/24689912e021

\section{KEY WORDS}

Integration, Infrastructure, Circulation, Waterway, Reprimarization. 


\section{INTRODUCCIÓN}

La infraestructura ha estado ligada en cada etapa económica a facilitar las actividades promovidas en el marco de las políticas predominantes y las formas de inserción internacional (Ferrer A., 1997; Rofman A. \& Romero L., 1997) de las economías de la región. La etapa llamada neoliberal (Arceo E. \& Basualdo E., 2006), implementada desde los años 70 y a partir de las dictaduras militares, se caracteriza por el quiebre del modelo desarrollista, la distribución regresiva del ingreso, la desindustrialización y las privatizaciones, acentuando la fragmentación socioterritorial y el impulso a actividades ligadas a la explotación de recursos naturales para exportación y a la valorización financiera.

En los albores del siglo XXI se avanza con una integración económica que promueve el libre comercio intra-regional como forma de ampliar mercados, escalas de explotación de recursos y áreas de actuación de las transnacionales, favoreciendo la creación de espacios transfronterizos para la acumulación del capital (Jessop, 2004). La infraestructura al servicio del modelo exportador ya no tiene escala nacional sino regional. Los organismos de crédito internacional la impulsan y fomentan en pos de la integración del territorio de América Latina con las nuevas redes del comercio internacional, sumando al Atlántico la conexión hacia el Pacífico.

En este contexto las grandes cuencas hidrográficas recobran su importancia estratégica como corredores de circulación de flujos hacia los puertos de exportación en ambos océanos. Organismos como la CAF (2016) o el BID (2000) pregonan que los ríos de la región podrían convertirse en auténticas rutas para transportar bienes y servicios e integrarse con autopistas y ferrocarriles y, al mismo tiempo, impulsar el desarrollo económico y la integración regional.

La cuenca hidrográfica del Paraná-Paraguay es fundamental para la integración sudamericana dado que está ubicada en el corazón geopolítico de la región. Representa el eje de penetración y transporte masivo de cargas de las economías de la Cuenca del Plata que desde los años 80 , junto con el avance de la frontera agrícola en los países que la conforman, generaron una demanda creciente de transporte en la hidrovía estimulando a Brasil, principal economía de la región, a revitalizar la circulación a través del mejoramiento de las condiciones de navegación de los ríos Paraguay y Paraná, en sintonía con Argentina, Paraguay y Bolivia (Dar Costa, 2011; Zugaib, 2006). 
Este trabajo es un aporte al estudio de la Hidrovía Paraguay-Paraná (HPP) como un corredor nodal en la ampliación de los circuitos espaciales de producción (Silveira, 2016; Arroyo, 1999; Santos 1979), aspecto imprescindible en los programas de integración en infraestructura regional. Está dividido en cinco apartados con el objetivo de: 1- problematizar la importancia de la ampliación de los circuitos espaciales de producción como un objetivo de la integración regional; 2- caracterizar los programas y proyectos existentes sobre la Hidrovía Paraguay-Paraná; 3- abordar el rol estratégico de la región en la expansión del modelo de reprimarización productiva imperante en América del Sur; 4- abordar la relación entre la Iniciativa para la Integración en Infraestructura Regional Suramericana (en adelante IIRSA) y las obras proyectadas para la Hidrovía; 5- conclusiones.

\section{LA INTEGRACIÓN REGIONAL COMO MECANISMO DE AMPLIACIÓN DE LOS CIRCUITOS} ESPACIALES DE PRODUCCIÓN

La dinámica global de circulación de flujos es central en la producción del territorio en América del Sur. La globalización neoliberal impuso la casi omnipresencia de la razón global en la producción del territorio potenciando aquellos puntos considerados estratégicos donde las técnicas, las informaciones y las normas globalizadas son más presentes y las densidades del medio técnico-científico-informacional son más altas (Silveira, 2011). La ampliación de los espacios de extracción, producción y circulación de mercancías, como también y de manera esencial del dinero y la información (Santos, 2004), fue generando en las últimas décadas una reconversión técnica en el transporte que permite una mayor fluidez de los territorios hacia el mercado global. Milton Santos (1996) advierte sobre las consecuencias de ese proceso de mundialización sobre los territorios:

Cuando las necesidades del proceso productivo llegan a todos los lugares, de manera directa e indirecta, se crean paralelamente selectividades y jerarquías de utilización con la competencia activa o pasiva entre los diversos agentes. En consecuencia, se plantea una reorganización de las funciones entre las diferentes fracciones del territorio. Cada punto del espacio adquiere entonces importancia, efectiva o potencial, que se desprende de sus propias virtualidades, naturales o sociales, preexistentes o adquiridas según intervenciones selectivas. Al mundializarse la producción, las posibilidades de cada lugar se afirman y se diferencian a nivel mundial. Dada la creciente internacionalización del capital y el 
ascenso de las empresas multinacionales, se observará una tendencia a la fijación mundial - y no nacional - de los costes de producción y a un equilibrio de las tasas de beneficios gracias a la movilidad internacional del capital (E. Mandel, 1978, pp. 187-188), al mismo tiempo que la búsqueda de lugares más rentables será una constante. (p.27)

Las diferencias geográficas y los recursos estratégicos en cada etapa histórico productiva, adquieren una importancia fundamental en la era de la globalización de la economía, ya que los actores hegemónicos en el sistema productivo mundializado pueden escoger el lugar ideal para una empresa concreta, lo cual requiere de intervenciones técnicas sobre el espacio: logística, infraestructuras de transporte, fuentes de energía, telecomunicaciones, etc., que generan las condiciones para una nueva apropiación en función de nuevos intereses. Tales especializaciones en la utilización del territorio significan un redescubrimiento de la naturaleza, una revalorización total del lugar que recibe un nuevo rol (Santos, 1996).

Es la deslocalización productiva una de las características fundamentales de la producción capitalista post crisis de los 70 y uno de los factores centrales es "la transformación de los territorios nacionales en espacios nacionales de la economía internacional y la exacerbación de las especializaciones productivas a nivel del espacio" (Santos, 1993, p. 7). Este proceso de fragmentación de la producción a escala global reproduce las históricas asimetrías entre los centros desarrollados y las periferias subdesarrolladas y es un factor central a la hora de caracterizar los proyectos de integración regional, sobre todo en lo concerniente a la integración física. La dinámica de los flujos adquiere entonces no sólo importancia económica sino también política en la producción del territorio.

El propósito de los proyectos de integración en infraestructura regional es suprimir todo obstáculo que dificulte la libre circulación de las mercaderías, de las informaciones y del dinero, a partir de las acciones técnicas y normativas que refuerzan el proceso de integración en paralelo a la transnacionalización del territorio. Las grandes obras de infraestructura, con un alto impacto en la morfología del espacio, son fundamentales en este sentido. Mónica Arroyo (1999) describe de la siguiente manera los efectos de este proceso:

Los circuitos espaciales de producción -en particular, los círculos de cooperación 
en el espacio- se extienden, aumentan su escala, organizan una trama de relaciones más allá de las fronteras nacionales. El tipo de jerarquías y selectividades creadas en el territorio se relaciona directamente con ese fortalecimiento de los flujos, ya que ellos se materializan en redes apoyadas sólo en ciertos puntos del territorio. Las grandes compañías financieras, de comercio internacional, de telecomunicaciones, de transporte se organizan en forma de red a escala mundial, atravesando los territorios nacionales con diferentes grados de intensidad y de articulación. Este movimiento produce una alteración, algunas veces muy profunda, en la división regional del trabajo preexistente y, por lo tanto, en la relación entre los actores locales. (p.7)

El metabolismo del capital se acelera a partir de este complejo entramado de redes donde la multimodalidad juega un rol central permitiendo que una misma carga pueda ser transportada por múltiples vías hasta llegar a destino, reduciendo tiempos y obstáculos a la circulación (Barreda, 2005). La técnica hace homogénea no sólo las pautas productivas sino también las formas de circulación. "El mundo actual exige fluidez, para la circulación de ideas, mensajes, productos o dinero, lo cual interesa a los sectores más dinámicos de la sociedad. Esa fluidez está basada en las redes técnicas, que son soportes de la competitividad" (Santos, 1996, p. 218). Se construyen objetos que transforman los lugares y las acciones con el fin de buscar la fluidez, como es el caso de la construcción de oleoductos, gasoductos, vías férreas, canales, autopistas, puertos, aeropuerto, puentes, túneles, etc. obras que permiten la interconexión multimodal del territorio, aumentando las ganancias de los principales actores económicos, a partir de la reducción de los costos de circulación.

Wilmsmeier (2015) destaca la importancia de la movilidad de la siguiente manera en un informe de la CEPAL:

La movilidad, en referencia al movimiento de personas, mercancías e información, ha sido un facilitador clave para la integración de las regiones y localidades en la economía mundial. Ésta se realiza por diversos medios y elementos constitutivos y, por lo tanto, se pueden diferenciar por el propósito, significado y competencias. Aspectos constitutivos de la movilidad son también la infraestructura física, las características de los servicios y los marcos institucionales que son quienes la facilitan. (p. 12) 
La buscada ampliación de los circuitos espaciales, definida por la movilidad de los flujos pero materializada en corredores regionales multimodales con un fuerte impacto en el ordenamiento territorial, trae como resultado el surgimiento de regiones económicas que exceden los límites tradicionales del Estado. En Suramérica la construcción de nuevas redes y de infraestructura tiene una escala regional y responde a las necesidades de actores que sobrepasan en su accionar los límites territoriales del Estado. Esto se expresa en el surgimiento de regiones transfronterizas (Jessop, 2004) que muchas veces se corresponden con organizaciones supranacionales preexistentes o en formación (MERCOSUR- UNASUR) y otras se relacionan directamente con los flujos de recursos, bienes, capitales, servicios, información, etc. preexistentes, actuales o futuros. Al respecto Jessop plantea:

Las transacciones transfronterizas basadas en proximidades $y$ complementariedades geográficas siempre han existido a niveles regionales y sub-regionales, a menos que sean prohibidas por los Estados en razón de preocupaciones políticas, de seguridad o económicas. Pero las regiones transfronterizas han proliferado siguiendo el declive del proteccionismo, el auge del neoliberalismo, el fin de la guerra fría, y los compromisos políticos hacia la integración regional. Las regiones transfronterizas se han convertido en objetos específicos de políticas y no solo en territorios económicos espontáneos y naturales. En este sentido, representan formas específicas de innovación en relación con el espacio, el lugar y la escala. Ellas implican la producción de nuevos tipos de lugares o espacios para la producción, para el desarrollo de servicios, para el trabajo y para el consumo. Están vinculadas con nuevos métodos de producción de lugares o espacios para crear ventajas específicas en la producción de bienes y servicios y ofrecer nuevas estructuras regulatorias, infraestructuras, nuevas economías de escala, mercados de trabajo, etc. (2004, p.32)

Los proyectos de integración regional en la Suramérica reciente han estado condicionados profundamente por las dinámicas globales de circulación de flujos. Estas dinámicas y las necesidades del capital de tender redes en los territorios fueron plasmadas desde el año 2000 en la IIRSA con una cartera de casi 600 proyectos de infraestructura en 9 ejes multinacionales con un fuerte impacto en la morfología del espacio regional. 
En este sentido la dinámica de los flujos y las diferentes escalas en que estos se desarrollan, con flujos hegemónicos y periféricos, es un factor muy importante a la hora de explicar el rol de los territorios de la región en la economía internacional, es un componente esencial para dilucidar las lógicas y las dinámicas de acumulación capitalista en tiempos de globalización. Ello obliga a desarrollar un análisis de escalas (García, 2006) que le otorgue centralidad a la relación entre las dinámicas globales, la infraestructura regional y el impacto local de las obras y los modelos primarizados.

Es posible inferir a partir del análisis de la circulación de recursos, productos y capitales, cuáles son las regiones privilegiadas por las grandes empresas, qué es lo que se extrae o se produce y cuáles son los principales destinos entre otros factores. También permite identificar cuáles son las condiciones indispensables para su desenvolvimiento: un marco de desregulación que desintegre las barreras legales a la libre circulación y un complejo de infraestructura que supere las barreras físicas son dos de estas condiciones.

Tomando como eje de estudio esas dos condiciones para la circulación, en el siguiente apartado se abordarán los acuerdos de integración regional vigentes y las obras de infraestructura proyectadas sobre la Hidrovía Paraguay-Paraná por ser considerada un territorio estratégico para el actual patrón de inserción de las economías de la región en la economía internacional y por ser una vía de articulación territorial central en la integración suramericana.

\section{El PROgRama de la HidRovía PaRAguay-ParanÁ: ANTECEdentes Y CARACTERÍSTICAS}

Las cuencas hidrográficas constituyen recursos de significativa importancia para la integración y el desarrollo regional, siendo un factor dinamizador del comercio nacional e internacional. La idea de avanzar en la integración de los ríos suramericanos es de larga data: Humbolt y Bompland hacia el 1800 planteaban la unión de las cuencas del Orinoco y el Amazonas. Al respecto Saguier (2015) argumenta:

La utopía de integrar las cuencas hidrográficas del Orinoco, del Amazonas y del Río de la Plata comenzó muy lentamente. Se inició con la aventura expedicionaria del bandeirante portugués Antonio Raposo Tabares (que violaba la línea fronteriza del Tratado de Tordesillas), a través de los ríos GuaporéMadeira hasta la boca o estuario del Amazonas luego de producida la rebelión 
de Portugal contra la dominación Habsburga (1648); y siguió con la llegada al Ecuador del hijo de la llustración Francesa, el explorador Charles Marie de La Condamine en 1735; y con los descubrimientos en Venezuela del naturalista alemán Alexander von Humboldt en 1799 [...] quien habría querido emular esos antecedentes históricos y remontando el Orinoco y las bifurcaciones del Casiquiare, en la Amazonía, descubrió la función natural de este último (esta expedición fue repetida dos siglos más tarde por otro alemán Klaus Reckling). En un intento de extender sus investigaciones hidrográficas (Del Orinoco al Amazonas. Viaje a las Regiones Equinocciales del nuevo continente), Humboldt envió a su socio y colega, el botánico y naturalista francés Aimée Bonpland, al Paraguay, para que explorara la integración fluvial de la cuenca del Plata y el Alto Paraguay con el Río Amazonas. (p.4)

En las últimas décadas la imbricada relación entre las hidrovías y la integración regional fue analizada de manera constante a través de diversos estudios, proyectos y reuniones académicas, pero en materia de publicaciones es de destacar el informe de la CAF "Los ríos nos unen. Integración Fluvial Sudamericana" de 1998 y la "Evaluación sobre los principales puertos de Sudamérica: análisis institucional, legal, de ingeniería, Ambiental y económico para el desarrollo de las obras en la Hidrovía ParaguayParaná" del año 2001.

Con respecto a esta Hidrovía, (en adelante HPP) constituye un espacio geopolítico de vital importancia para los países que la componen, utilizada desde épocas antiguas como canal de comercialización, transporte y comunicación entre los territorios, constituyéndose en la actualidad en una plataforma para la inserción de la región en el contexto internacional y regional (Bono, 2014).

En términos de integración política de los países que componen la Cuenca del Plata y tienen soberanía sobre la HPP, es importante remarcar la existencia de un Programa para el desarrollo de la Hidrovía cuyos antecedentes se remontan al año 1969 cuando se firmó el Tratado de la Cuenca del Plata. Este instrumento busca el desarrollo armónico de la región y la integración física de la Cuenca y sus áreas de influencia, mediante el perfeccionamiento de las interconexiones viales, ferroviarias, fluviales, aéreas, eléctricas y de telecomunicaciones, entre otros puntos. 
El posterior Tratado de las Leñas, del año 1992, claramente influido por el contexto de regionalismo abierto imperante en la región (CEPAL, 1994), es fundante de un ambicioso proyecto de integración para los países de la Cuenca, postulando que los Estados Parte están al momento de la firma del acuerdo:

Persuadidos que la Hidrovía Paraguay-Paraná [...] constituye un factor de suma importancia para la integración física y económica de los Países de la Cuenca del Plata [...]; crea una comunidad de intereses que debe ser apoyada en forma adecuada, eficaz y mancomunada, basada en la igualdad de derechos y obligaciones de sus países ribereños [y por lo tanto se encuentran], decididos a crear las condiciones necesarias para concederse mutuamente todas las facilidades y garantías posibles a fin de lograr la más amplia libertad de tránsito fluvial, de transporte de personas y bienes y la libre navegación. (p. 1)

Este tratado propicia entre los cinco Estados Partes el perfeccionamiento de las interconexiones viales, ferroviarias, fluviales, aéreas, eléctricas y de telecomunicaciones, entre otros puntos, buscando el desarrollo armónico de la región, complementando las iniciativas de los cancilleres que declararon en 1987 como de vital interés el desarrollo del sistema fluvial de los ríos Paraná y Paraguay y, en septiembre de 1989, crearon el Comité Intergubernamental de la Hidrovía (en adelante $\mathrm{CIH}$ ) iniciando dicho órgano sus actividades en 1990 (Bono, 2014). Al respecto Laura Bono (2014) aclara las incumbencias del Comité:

El ClH es el órgano encargado de coordinar, proponer, promover, evaluar, definir y ejecutar las acciones identificadas por los Estados Partes respecto del Programa de la HPP, así como gestionar y negociar previa conformidad de las autoridades nacionales, los acuerdos de cooperación técnica para el desarrollo del sistema fluvial involucrado, constituyéndose asimismo en foro de entendimiento para los asuntos relacionados con el proyecto. A su vez el $\mathrm{CIH}$ es el órgano político del Acuerdo de Transporte Fluvial por la HPP, suscripto en el Valle de las Leñas en 1992, el cual ha entrado en vigor en febrero de 1995. (p. 86)

En el marco del Acuerdo se establecieron acciones y obras necesarias para alcanzar el objetivo de mejorar las condiciones de navegación en la Hidrovía, permitiendo la utilización de la misma durante las 24 horas diarias los 365 días del año. El programa 
plantea la readaptación de la flota existente y la mejora en la infraestructura portuaria, acorde con las necesidades comerciales de la región. Bono (2014) plantea que el cronograma se estructuró de la siguiente manera:

Una primera parte contempla las obras de infraestructura propiamente dichas y de inversión entre las cuales puede citarse: a) Implementación y mantenimiento de obras y servicios en la vía navegable, b) Sistema portuario, incluyendo obras, equipos y mantenimiento y c) Flota, incluyendo adquisición y mantenimiento de la existente; y una segunda etapa que contempla el estudio vinculado a las cuestiones jurídicas, institucionales y administrativas, las cuales se han convertido en uno de los principales inconvenientes del Programa. (p. 84)

Es importante remarcar que el Programa estuvo centrado exclusivamente en la promoción de obras de infraestructura que mejoren la navegabilidad de los ríos que componen la Hidrovía y que, en ese sentido, desde la primera reunión del ClH ya se definieron las prioridades para cada uno de los Estados Parte. Estas prioridades fueron complementadas desde el año 2000 con la cartera de proyectos de IIRSA y proyectos de instituciones internacionales como la CAF, que anunció en el 2016 el lanzamiento de un Programa Regional para el desarrollo de las Hidrovías de Sudamérica donde contemplan muchas de las obras incorporadas en estas iniciativas precedentes para los ríos Paraná, Paraguay y Uruguay.

\section{HIDROVÍA, INTEGRACIÓN REGIONAL Y REPRIMARIZACIÓN. TERRITORIOS EN DISPUTA}

Se ha hecho referencia ya a la importancia de la Cuenca del Plata en la integración regional y el valor estratégico que la HPP ha tenido siempre para América del Sur como medio de transporte y como factor de integración económica, política y cultural. Esta importancia se acrecienta en las últimas décadas dada la valorización económica de los territorios como consecuencia del avance del agronegocio, y otras actividades extractivas en los países que la componen, proceso que ha generado una profunda reprimarización productiva (Bolinaga \& Slipak, 2015).

El crecimiento de la producción regional de commodities hace indispensable en la expansión de la rentabilidad de las grandes empresas, actores centrales de este modelo, optimizar las condiciones de navegación y adecuar la infraestructura portuaria. Ambos aspectos están contemplados en los planes y programas de infraestructura y logística previstos para los ríos Paraná y Paraguay y se vienen 
desarrollando desde la creación del $\mathrm{ClH}$. El tráfico actual en la Hidrovía es casi exclusivamente descendiente y se concentra entre Corumbá y Río de la Plata, bajo la forma de convoyes que transportan granos y minerales (Capra, 2003). Un reciente informe de la Fundación Instituto de Desarrollo Regional de Rosario (2018) aporta precisión sobre los calados afirmando que "El Sistema de Navegación troncal del Paraná está conformado por una sucesión de tramos rectos de distinta longitud unidos por curvas, la profundidad garantizada actualmente es de 34 pies entre el océano y Puerto San Martín y 25 pies para la sección Puerto San Martín- Santa Fe" (pp. 86-87).

En Rosario y los puertos aledaños (ROSAFE) se ha generado el cluster de producción y exportación de soja más importante de la región y uno de los más importantes del mundo (Zuberman, 2012), con una vasta infraestructura multimodal. Con respecto al tráfico comercial el informe de la Fundación Instituto de Desarrollo Regional de Rosario (2018) titulado "Presente y futuro del transporte por la hidrovía ParaguayParaná", precisa que:

A través de la Hidrovía y su infraestructura se encamina, principalmente, la comercialización internacional de la producción de granos y derivados agroindustriales de la Argentina, Paraguay, Bolivia y parte de la producción del Estado de Mato Grosso $(\mathrm{Br})$. Esta región extendida tiene un área sembrada de más de 32 millones de hectáreas, con una producción de soja de unos 96 millones de toneladas en la campaña 2015/16 equivalentes a la producción total de Brasil que es el segundo productor de soja del mundo. (p.56)

El volumen transportado de soja el año 2016 fue de 47,7 millones de toneladas distribuidas de la siguiente manera: Argentina 39,7 millones, Bolivia 3,9 millones, Paraguay 3,8 millones y el Mato Grosso 0,33 millones; a esto se deben agregar los tráficos ascendentes de insumos para la producción agrícola. En este sentido, si bien, como ya se ha hecho referencia, los principales productos de exportación transportados por la Hidrovía son los granos como soja, maíz, trigo, cebada, colza, sorgo, arroz y maní, junto a derivados como harinas, pellets o aceites, en las últimas décadas y, en menor medida, otra carga importante es el mineral de hierro cuyo origen es de los yacimientos Mutún en Bolivia y de Urucum en Brasil. Es significativo destacar además, en tanto valor estratégico del tránsito fluvial, que para Paraguay como para Bolivia la Hidrovía es una vía de acceso al Océano Atlántico de gran importancia por ser países mediterráneos. 
En relación con lo antes descrito, varios informes de instituciones y organismos especializados como la CEPAL (2014), BID (2015), entre otros, hacen referencia a la importancia de la optimización en la infraestructura de transporte en la región para mejorar la competitividad y las ganancias de las empresas. Ricardo J. Sánchez (2017), Jefe de la Unidad Servicios de Infraestructura de División de Recursos Naturales e Infraestructura de la CEPAL, hace hincapié en diversos estudios sobre la correspondencia entre la inversión en infraestructura y el desarrollo económico de un determinado territorio:

Las inversiones en infraestructura contribuyen a la reducción de los costos y al aumento de la productividad. La hipótesis central es que las inversiones en infraestructura de transporte son condición necesaria para el desarrollo productivo de una región, en particular aquella ligada al comercio exterior, como los puertos y las vías navegables, quedando ésta demostrada al observarse una correspondencia positiva entre la evolución de las prestaciones de servicios portuarios e hidroviarios (con menores costos y tiempos operativos, mayor confiabilidad y nuevos servicios), con la expansión de la frontera agrícola, el crecimiento de la productividad y la producción agrícola y su industrialización. A su vez plantea que la provisión de una infraestructura con menores costos y tiempos operativos y mejores y más confiables servicios, depende no sólo de la infraestructura física sino también de las condiciones de mercado creadas por las políticas del transporte y la regulación económica del mismo, como también de las características del mercado de operación del transporte (p. 4)

Las mejoras en la provisión de infraestructura en el Paraná medio e inferior han posibilitado la ampliación del hinterland de los puertos localizados al sur de la provincia de Santa Fe (alrededor de Rosario, San Lorenzo y San Martín), extendiéndose a zonas tales como el norte argentino, ciertas áreas de Brasil, Bolivia y Paraguay, el alto Paraná y algunas zonas de producción minera (de la cordillera de los Andes) que tienen como salida a la mencionada región portuaria, además de la tradicional zona de influencia de los puertos del área sur del Río Paraná.

El incremento en inversiones en infraestructuras se observó en particular en el desarrollo de las prestaciones portuarias e hidroviarias, sobre todo en la zona de ROSAFE que han apoyado, en un contexto de aumento de la demanda (sobre todo del mercado asiático) y los precios internacionales, el desarrollo de la región agrícola 
antes mencionada y su productividad, especialmente de soja. En la actualidad los diferentes actores que participan del agronegocio en la región impulsan nuevas obras de infraestructura que faciliten la navegación continua en la Hidrovía, para lo cual se visualiza como de importancia vertebral la profundización del calado y la optimización de la infraestructura portuaria (de los 18 puertos existentes en la zona de Rosario 14 están en manos extranjeras).

Una hipótesis central, en relación a los proyectos de integración física hegemónicos, es que iniciativas como IIRSA impulsan un complejo entramado de infraestructuras con una visión netamente productivista; infraestructuras verticalizadas que responden a las necesidades de las grandes corporaciones desconociendo en muchos casos criterios y problemáticas sociales y ambientales. En el caso de la Hidrovía, su diversidad morfológica, sobre todo en el tramo superior sobre el Rio Paraguay, obliga a realizar obras con mayor impacto dado la dificultad natural para el tránsito de barcos de gran porte. Al respecto Stancich (2006) plantea:

Hay zonas del río Paraguay, por ejemplo, que son muy delicadas; muy cerca del Pantanal algunos expertos dicen que, para que el ecosistema pueda soportar la navegación tendría que estar pasando sólo un tren de barcazas por semana, de manera tal que la naturaleza pueda restablecer todo lo que es el ecosistema acuático sin que la navegación lo afecte. Pero con la carga que hay proyectada por día podrían estar pasando 8 o más trenes de 20 barcazas cada uno. Todo esto no está profundizado en los estudios; creo que vale la pena ponerlo en debate y analizar mucho más. Cuál va a ser el impacto sobre la pesca, sobre la calidad del agua, sobre los sedimentos, la selva en galería que hoy sigue siendo destruida por la navegación en tramos del río Paraguay en el Pantanal. (p.4)

Diferentes estudios sobre el efecto ambiental de las obras sobre la Hidrovía como los de Moiraghi de Pérez (2001), Coria (2010) o Adámoli (2016), remarcan que el aumento del tránsito fluvial junto al desarrollo de mega obras de infraestructura que lo hacen posible alteran el equilibro natural del sistema generando perjuicios inevitables. Moiraghi de Perez (2001) plantea que:

En toda la Cuenca del Plata existe una armonía natural, el río Paraná crece en verano y no coincide con las crecidas del río Uruguay que crece en invierno y baja en verano; el río Iguazú que viene de la misma naciente del río Uruguay, 
también crece en invierno, tiene un efecto compensador con el Paraná y el Paraguay. El Pantanal ejerce un efecto retardador evitando que las grandes lluvias estivales se canalicen automáticamente y repercutan a los quince días, haciendo que se sientan más o menos cuatro meses después. (p.1)

Sin embargo, la autora plantea que ese equilibrio se va modificando paulatinamente dado que el aumento de la navegación y la mayor magnitud de los buques, así como los convoyes de barcazas producen erosión costera y consiguiente pérdida de la vegetación de las orillas y destrucción de la llamada "selva marginal" hábitat de aves y otros animales. Para Moiraghi de Perez:

El río Paraguay entre Cáceres y Corumbá, es un tramo crítico dentro del proyecto hidrovía a causa de la riqueza ecológica, complejidad y fragilidad del Pantanal, y por la regulación del flujo de agua a través de todo el sistema dado el rol de "esponja" que juega la zona. Se han determinado algunos tramos para asegurar dimensiones mínimas de navegación con canalización, rectificación del curso del agua y construcciones para el control del agua. Además habrá que prevenir la contaminación de las aguas por el aumento de la navegación y porque a partir de la urbanización creciente los ríos podrían convertirse en propagadores de enfermedades transmisibles provenientes de las regiones selváticas y de los desechos industriales cada vez más crecientes a lo largo de la cuenca. (p. 3)

Coria (2008) aporta otros datos significativos en relación al impacto ambiental de la modificación de la Hidrovía: "la ampliación, profundización y rectificación de canales puede alterar el régimen hídrico del río Paraguay, aumentando la velocidad de traslado de las crecidas, aumentando los riesgos de éstas en todo el curso de este río" (p. 1). El autor afirma que las obras afectarían los sistemas de humedales incluyendo el Gran Pantanal del Alto Paraguay, lo que perjudicaría su condición de esponja natural estabilizadora de los flujos del río:

Las modificaciones en el canal del río Paraguay pueden producir el desecamiento de partes de los esteros y bañados a lo largo de sus planicies de inundación, incluyendo porciones del Gran Pantanal del Alto Paraguay; los esteros del Chaco, San Pedro y Ñeembucú en el Paraguay; los sistemas de 
bañados del Yvera (Iberá) en Corrientes (Argentina) y otros sistemas de humedales. (p 2)

Estos impactos ambientales complejizan aún más los conflictos sociales desarrollados como consecuencia del desarrollo de un modelo productivo/extractivo altamente concentrado donde son las empresas transnacionales los principales actores. Sólo en Argentina, según un informe sobre conflicto rural del Ministerio de Agricultura del año 2014, existen al menos 9,3 millones de hectáreas de campesinos e indígenas que son pretendidas en la mayoría de los casos por grandes empresas. La tierra en disputa equivale a 455 veces la superficie de la Capital Federal, son 857 casos y afecta a 63.843 familias. El $64 \%$ de los conflictos comenzó en las últimas dos décadas y se localizan mayoritariamente en parte de la Cuenca del Plata.

La integración en infraestructura a partir del desarrollo del Programa de la HPP, al igual que toda la infraestructura regional comprendida en IIRSA-COSIPLAN, se encuentra ante una encrucijada y un debate fundamental. La inversión en obras de infraestructura verticalizadas es importante en la expansión de las actividades más dinámicas del modelo de inserción de Suramérica en el comercio internacional, pero estas obras profundizan, por un lado, los impactos ecológicos y sociales del agronegocio y, por otro, como lo demuestra lo antes expuesto y se profundizará en el apartado siguiente, consolidan un desarrollo dependiente de las demandas del comercio internacional y de las inversiones de las grandes empresas transnacionales.

\section{IV.IIRSA-COSIPLAN Y LA HIDROVÍA PARAGUAY-PARANÁ}

La HPP representa el $23 \%$ de la superficie del continente de América del Sur. Se estructura en torno a las cuencas de los Ríos Paraguay, Paraná, y Uruguay, todas tributarias de la gran Cuenca del Río de la Plata. Es una de las vías fluviales navegables más importantes del mundo con una extensión aproximada de $3.442 \mathrm{~km}$ comprendidos de Norte a Sur desde Puerto Cáceres (Brasil) hasta Puerto Nueva Palmira (Uruguay).

El eje es el segundo más vasto de los 9 proyectados en IIRSA-COSIPLAN con el $20 \%$ de la superficie de Suramérica $\left(4.201 .862 \mathrm{~km}^{2}\right)$ y el segundo con mayor porcentaje de PBI de la región, con el 34\% (1.491.032 millones de dólares). Es además el tercero con más población, con el $29 \%$ de la población total del continente, 119.035.634 habitantes (IIRSA - COSIPLAN, 2017). 


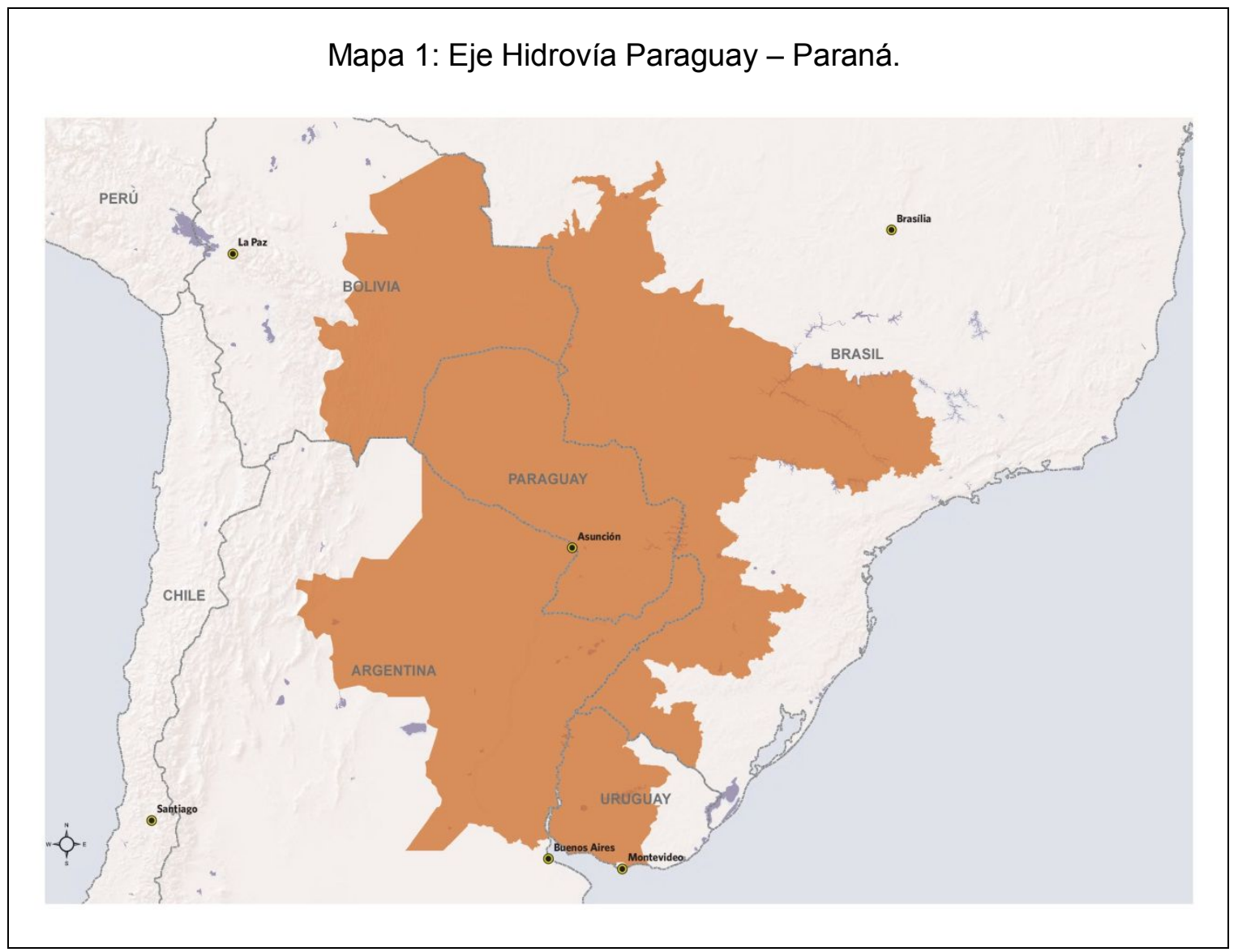

Fuente: IIRSA-COSIPLAN, 2017

Los países que integran la HPP planifican inversiones por 7.534 millones de dólares en 84 proyectos de integración física. Este es el segundo eje con mayor cantidad de proyectos de la Cartera, después del MERCOSUR-Chile; sin embargo, ocupa el séptimo lugar en montos de inversión estimada. Esto se debe a que las obras fluviales demandan, en comparación con el carretero y otros sub-sectores, menores inversiones (IIRSA - COSIPLAN, 2017)

El mayor impacto buscado por la IIRSA en las obras planificadas para la Hidrovía es mejorar la navegabilidad de los ríos que la componen y la conexión interregional, para conectar las economías con los puertos de salida tanto fluviales como los ubicados en el Atlántico y en el Pacífico. La reducción de los tiempos de transporte es significativa, pues pasará de 36 a 16 días para el trayecto Corumbá - Río de la Plata- Corumbá $(5.500 \mathrm{~km})$. Asimismo, la posibilidad de conectar fluvialmente Sao Paulo con Buenos Aires, los dos grandes centros económicos de la región, se abre con el tramo ParanáTieté, considerado por IIRSA como un proyecto-ancla (Ceceña, Aguilar, Motto, 2007). 
En la Argentina la zona portuaria de Santa Fe es de gran importancia para el desarrollo de la Hidrovía ya que es allí donde ésta se articula con el Corredor Bioceánico Central (interoceánico) que conecta Brasil con el Pacífico. Esta posición geoestratégica la convierte en un punto logístico de carga, descarga, acopio y transporte de bienes en dirección Norte-Sur y Este-Oeste. Es un corredor esencial para los planes de integración regional y reordenamiento territorial de Suramérica, un eje articulador de otros ejes, sobre todo del Corredor Bioceánico que permite trasladar la producción de Brasil (principal economía de la región) para su exportación por el Océano Pacifico rumbo, sobre todo, al mercado asiático.

El siguiente mapa ilustra la posición estratégica de Santa Fe en la HPP si se toma en cuenta la articulación con un eje interoceánico que, a través del Paso de Aguas Negra, obra que está proyectada su finalización en 2022, permitirá reducir los tiempos de circulación Este - Oeste de las mercancías. Este corredor es de mucha importancia para la producción minera del Oeste Argentino pero también para la producción agroindustrial de la Cuenca del Plata y como corredor de acceso de los productos de Bolivia, Paraguay, Uruguay y Brasil al Océano Pacifico.

Mapa 2: Corredor Bioceánico Porto Alegre - Santa Fe - Coquimbo 


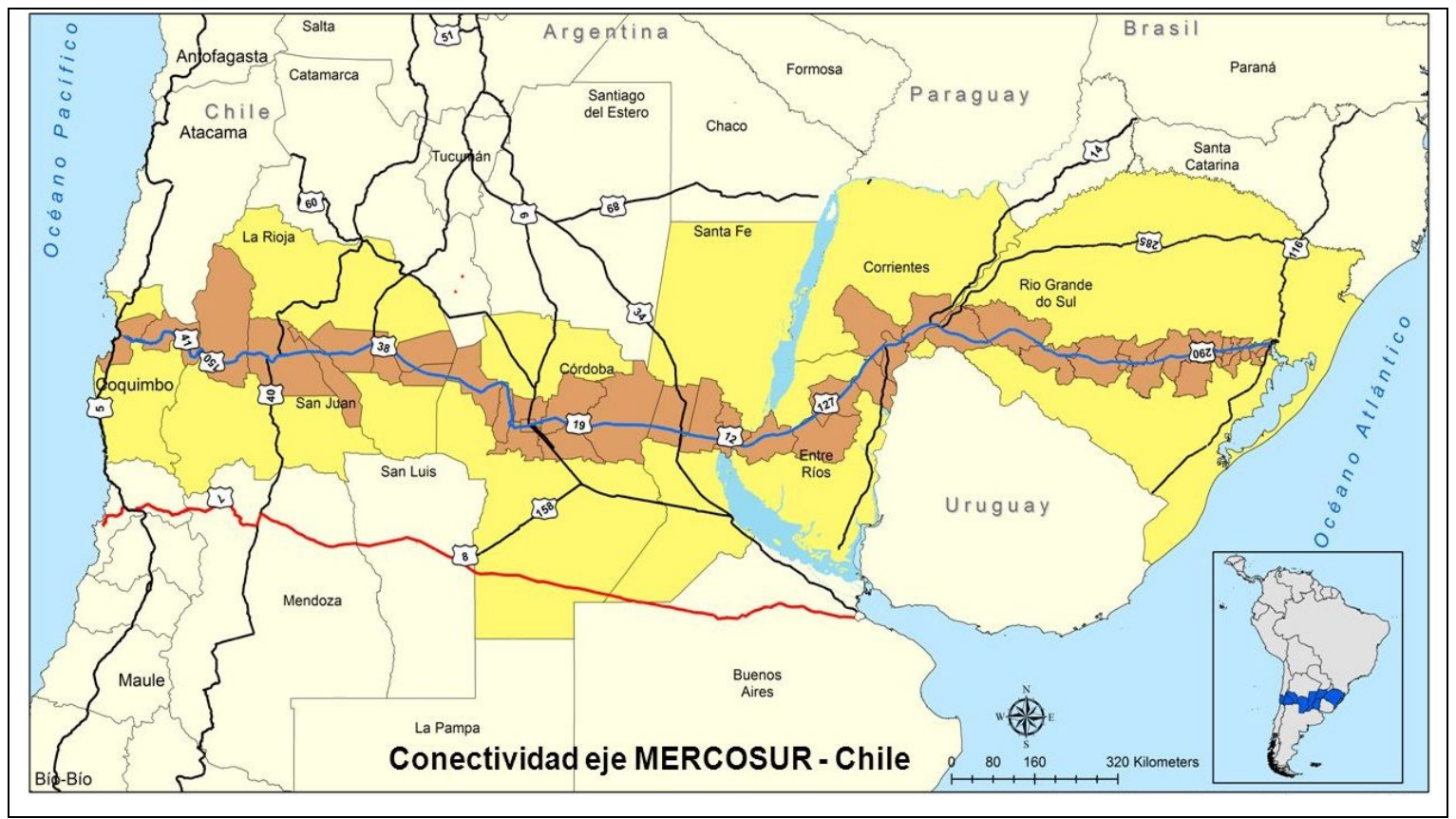

Fuente: Pinto Cornejo, Proyecto FIC-R Universidad Católica del Norte - Gobierno Regional de Coquimbo, 2018

La articulación entre corredores Norte-Sur y Este-Oeste es significativa dado que, en términos de proyecciones, suscribiendo al análisis de Zuberman (2012), los países pertenecientes a la Cuenca del Plata, es decir Brasil, Argentina, Paraguay y Bolivia en forma conjunta, conforman el mayos aglomerado de producción de soja a nivel mundial desde hace casi una década. Esto es central porque obliga a una planificación estratégica que le de centralidad a la circulación y sobre todo a la articulación de los corredores internos con los puertos en ambos océanos, significando un condicionante para las formas dominantes de integración física regional.

El modelo de re-primarización además, seguido por los países de la región tras la recomposición del ciclo del capital a inicios del siglo XXI, es hegemonizado por las grandes corporaciones transnacionales que tienen un alto impacto sobre las políticas de planificación regional de los Estados, sobre todo por la capacidad de éstas de atraer divisas en un contexto de restricción interna por parte de los países de la región. En la HPP operan empresas transnacionales como Cargill, Monsanto, ADM, Bunge, Dreyfus, etc., que impulsan nuevos usos del espacio geográfico desplazando los tradicionales por la siembra de monocultivos transgénicos que aportan mayor rentabilidad dado el aumento de la demanda internacional de estos commodities. 
En este sentido y en el marco de investigaciones realizadas sobre la HPP y su importancia en la integración regional, entre las que se encuentra el presente trabajo, se entrevistó a Juan Carlos Venesia, de reconocida trayectoria en el estudio y promoción de la infraestructura regional, actualmente Director Ejecutivo del "Programa de Infraestructura Regional para la Integración" y Director del "Programa Santafesino para el Desarrollo de la Hidrovía Paraguay-Paraná". Venesia, uno de los actores claves en los últimos 20 años en los actuales proyectos de desarrollo fluvial en Argentina, enfatiza, tal como se viene haciendo en esta investigación en el peso de las dinámicas globales en las infraestructuras locales, el rol de los puertos, y la importancia de un enfoque que adopte una perspectiva geopolítica:

Los grandes fenómenos de infraestructura dependen de los abordajes desde donde se desarrollan: si uno lo ve desde una ciencia dura, ingeniería, puede tener un tipo de respuesta, pero desde la ciencia social o desde un abordaje económico la respuesta de la ingeniería no alcanza. Fundamentalmente en situaciones como el análisis de la Hidrovía Paraguay-Paraná y los grandes fenómenos de conectividad hay que verlos en una escala cuasi geopolítica (J. C. Venesia, entrevista personal, 20 de septiembre de 2018).

Venesia hace hincapié en que la infraestructura regional y la integración física están condicionadas por un cambio en la dinámica de las exportaciones, que sin modificar su composición primaria en las últimas décadas fue incrementado el peso de la exportación por el occidente de la región hacia el mercado asiático:

Cuando uno ve situaciones como las de la Hidrovía debe ver la escala supra para después ver los impactos locales, no es lo local lo que impone la dinámica. Históricamente la Argentina en el modelo de la división internacional del trabajo del siglo XIX asumió un rol agroexportador, un modelo de exportación de graneles, de productos primarios, mucho no hemos cambiado más allá que el sistema mundo del que habla Wallerstein ha girado el eje del Atlántico en términos comerciales a tener mayor preponderancia el eje Pacífico (J. C. Venesia, entrevista personal, 20 de septiembre de 2018).

Este cambio geopolítico es central en el desarrollo de proyectos de integración física en la región, dado que la guerra comercial desatada en las últimas décadas entre los principales actores del sistema internacional tracciona los mercados de los países de 
la periferia y obliga a desarrollar proyectos de infraestructura, en integración regional, acordes a esos requerimientos globales.

América del Sur se encuentra ante una nueva encrucijada en sus iniciativas de integración en infraestructura: o continúa con proyectos conniventes con los capitales transnacionales y las demandas de los principales actores del sistema internacional, como lo fue IIRSA/COSIPLAN en las últimas dos décadas, profundizando un capitalismo dependiente $\mathrm{o}$, aprovechando las ventajas geográficas y productivas, retoma proyectos de regionalismo autónomos que vayan acompañados por planes integrales de desarrollo territorial e infraestructura potenciando la complementariedad de las economías regionales en equilibrio con las demandas exógenas.

\section{CONCLUSIONES}

La ampliación de los circuitos espaciales de producción y circulación en América del Sur ha generado en las últimas décadas el surgimiento de lo que se ha denominado regiones transfronterizas (Jessop, 2004), espacios de acumulación del capital que exceden los límites del Estado para lo cual se requieren proyectos de integración regional, sobre todo en el área de infraestructura, que den cuenta de una movilidad de flujos que trascienden las fronteras del Estado y que tienen como objetivo satisfacer demandas exógenas. Hecho evidenciable, como se ha abordado en este trabajo, en la expansión de la frontera sojera en la Cuenca del Plata.

En este sentido, en la visión dominante sobre la integración en infraestructura regional, encarnada en la IIRSA pero materializada también en el programa de la HPP en los últimos años, hay un énfasis manifiesto en la formación de un área de libre comercio regional y en el estrechamiento de lazos con el mercado global ganando importancia la edificación de corredores de exportación que funcionan concretamente como enlaces hacia las costas. En esta perspectiva la provisión de infraestructura debe desarrollarse en dos sentidos: libre comercio regional y conexión global (Zibechi, 2015).

Esta es la visión de IIRSA en sus casi dos décadas de desarrollo desde que fue pensada e ideada antes de la Primera Cumbre de Presidentes de América del Sur en Brasilia en el año 2000. Es importante recordar, en la contextualización del surgimiento de la iniciativa, que fue gestada en el marco de los debates por el establecimiento en la región de un Área de Libre Comercio de las Américas (ALCA) y pervivió en las diferentes correlaciones de fuerzas y momentos de las relaciones internacionales 
suramericanas hasta la actualidad. En IIRSA se promueve una interconexión eficiente, en términos de circulación, de la producción de la región en el espacio regional y en los mercados globales, reforzando la idea de complementariedad de la economía internacional y, por ende, las ventajas comparativas estáticas de los países en la división internacional del trabajo.

Se podría afirmar entonces como una primera conclusión que estos proyectos hegemónicos de integración física consolidan un desarrollo geográfico desigual al interior de la región y de ésta con la economía del mundo capitalista, donde el criterio que prima es el de la construcción de una infraestructura que refuerce los flujos actuales y potenciales de circulación, lo que refuerza también la heterogeneidad estructural en desmedro del desarrollo de los territorios menos favorecidos en términos productivos, generando además fuertes impactos sociales y ambientales, como se demostró en las obras proyectadas para la HPP.

La edificación de corredores bioceánicos, como el Corredor Interoceánico Porto Alegre-Santa Fe-Coquimbo, que en una visión de desarrollo regional e integración deberían promover la interconexión de las áreas interiores construyendo verdaderas redes de integración, apelando a las complementariedades regionales y la cooperación, están concebidos en realidad de forma subordinada a las lógicas y las dinámicas, por ende a las demandas, del mercado global, significando esto que fueron pergeñados de forma subordinada a la idea de una integración hacia afuera bajo la premisa del "regionalismo abierto" (CEPAL, 1994).

De esta manera es oportuno afirmar, como una segunda conclusión de este trabajo, que iniciativas como IIRSA no constituyen proyectos regionales de integración sino una cartera de obras de interconexión regional y sobre todo de articulación de los recursos estratégicos de la región con el mercado internacional, aspecto evidenciado en las características del tránsito y las obras promovidas en la HPP. Zibechi (2015) denominaría a esto "interconexión sin integración", es decir, un proyecto que interconecta zonas consideradas muy importantes para los actuales patrones productivos con los puertos de exportación en ambos océanos pero que no integra los diferentes territorios del subcontinente entre sí con el fin de potenciar su desarrollo (económico, productivo, social, ambiental, político) de una manera equilibrada. 
América del Sur se encuentra hoy frente a un nuevo debate paradigmático en la integración regional ante un renovado ciclo neoliberal que desarticuló la arquitectura de instituciones supranacionales construidas en los primeros años del siglo XXI, sobre todo la UNASUR. Esto abre interrogantes sobre el futuro de los programas de integración en infraestructura, fundamentalmente ante una profunda crisis económica en las principales economías de la región y un escenario político de conflictos abiertos.

\section{BiBLIOGRAFÍA}

Arceo, E. \& Basualdo, E. (Comps.). (2006). Neoliberalismo y Sectores Dominantes. Tendencias globales y experiencias nacionales. Buenos Aires, Argentina: CLACSO.

Arroyo, M. (1999). Notas sobre los cambios de final de siglo XX (A propósito de la globalización y su "irreversibilidad"). Revista Cátedra de la Facultad de Filosofía, Ciencias y Letras de Colatina, (2), 1-13. Recuperado de: https://www.educ.ar/recursos/buscar?q=Notas+sobre+los+cambios+de+final+de+siglo $+\mathrm{XX}+\% 28 \mathrm{~A}+$ prop $\%$ C3\%B3sito+de+la +globalizaci\%C3\%B3n+y+su+\%E2\%80\%9Cirrev ersibilidad $\% \mathrm{E} 2 \% 80 \% 9 \mathrm{D} \% 29 .+$

Banco Interamericano de Desarrollo. (2000). Un nuevo impulso a la integración de la infraestructura regional en América del Sur. Recuperado de: http://www.iirsa.org/Document?menultemld=5

Barreda, A. (2005). Geopolítica, recursos estratégicos y multinacionales. Pueblos, Revista de Información y Debate. Recuperado de: http://www.revistapueblos.org/old/spip.php?article311

Bericat, E. (1998). La integración de los métodos cuantitativo y cualitativo en la investigación social. Barcelona, España: Ariel.

Bidaseca, K. (Dir.) (2013). Relevamiento y sistematización de problemas de tierras de los agricultores familiares en Argentina. Buenos Aires, Argentina: Ministerio de Agricultura Ganadería y Pesca Recuperado de: http://www.ucar.gob.ar/images/publicaciones/Relevamiento $\% 20 y \% 20$ sistematizaci\%C3 \%B3n\%20de \%20problemas $\% 20$ de $\% 20$ tierra $\% 20$ de $\% 20$ los $\% 20$ agricultores $\% 20$ familiar es\%20en\%20Argentina.pdf

Bolinaga, L. \& Slipak, A. (2015). El Consenso de Beijing y la reprimarización productiva de América Latina: el caso argentino. Problemas del Desarrollo, 46(183), 33-58. https://doi.org/10.1016/j.rpd.2015.10.003

Bono, L. (2014). Los proyectos de infraestructura física en la región sudamericana y su relación con los procesos de integración comercial (2000 - 2010). El caso de la Hidrovía Paraguay-Paraná (Tesis de Maestría en Relaciones Internacionales). Recuperado de: http://www.iri.edu.ar/images/Documentos//tesis/tesis bono.pdf 
Capra, K. (2003). La Hidrovía Paraná Paraguay. Una alternativa a los puertos del Pacifico. Unidad de Análisis de Políticas Sociales y Económicas. Estado Plurinacional de Bolivia: UDAPE. Recuperado de: http://www.udape.gob.bo/portales html/Documentos\%20de\%20trabajo/DocTrabajo/20 $\underline{\text { 03/HPP1.pdf }}$

Comisión Económica Para América Latina y el Caribe (2014). La brecha de infraestructura económica y las inversiones en América Latina. Boletín FAL, (332). Recuperado

http://repositorio.cepal.org/bitstream/handle/11362/37286/Bolet\%C3\%ADn\%20FAL\%2 0332 es.pdf

Comisión Económica para América Latina y el Caribe. (1994). El regionalismo abierto en América Latina. La integración económica al servicio de la transformación productiva con equidad. Santiago de Chile, Chile: Naciones Unidas. Recuperado de: https://repositorio.cepal.org/bitstream/handle/11362/2140/1/S9481108 es.pdf

Congreso de la Nación Argentina. Ley № 24.385 (1994). Acuerdo de Transporte Fluvial por la Hidrovía Paraguay-Paraná (Puerto de Cáceres-Puerto de Nueva Palmira) suscripto con las Repúblicas de Bolivia, Federativa del Brasil, Paraguay y Oriental del Uruguay. Recuperado de: http://servicios.infoleg.gob.ar/infolegInternet/anexos/04999/774/norma.htm

Coria, I. (2008). Hidrovía Paraguay-Paraná: consecuencias ambientales. Simposio Ecoepisteme en IDICSO-USAL, Octubre. Argentina: FEPAI. Recuperado de: http://www.fepai.org.ar/Ecoepisteme/2008/Coria.pdf

Costa, D. (2011). La estrategia de la Integración. En D. Costa. (Comp.), América del Sur. Integración e Infraestructura. Río de Janeiro, Brasil: Capax Dei.

Ferrer, A. (2006). Globalización, desarrollo y densidad nacional: un abordaje a la experiencia de América Latina. En W. Ansaldi (Dir.). La democracia en América Latina: un barco a la deriva (pp 123-130). Buenos Aires, Argentina: Fondo de Cultura Económica.

Fundación Instituto de Desarrollo Regional de Rosario. (2018). Presente y futuro del transporte en la Hidrovía Paraguay-Paraná: perspectivas económicas de su ampliación. Rosario, Santa Fe: Fundación Instituto de Desarrollo Regional de Rosario.

García, R. (2006). Sistemas Complejos. Conceptos, método y fundamentación epistemológica de la investigación interdisciplinaria. Barcelona, España: Ed. Gedisa.

Iniciativa para la Integración de la Infraestructura Regional Suramericana -IIRSAConsejo Suramericano de Infraestructura y Planeamiento -COSIPLAN-. (2017). Cartera de Proyectos 2017. Buenos Aires, Argentina: COSIPLAN. Recuperado de: http://www.iirsa.org/admin iirsa web/Uploads/Documents/CARTERA DIGITAL.pdf 
Jessop, B. (2004). La economía política de la escala y la construcción de las regiones transfronteriza. Revista Eure, 29(89), 25-41. Recuperado de: https://scielo.conicyt.cl/pdf/eure/v30n89/art02.pdf

Moiraghi de Pérez, L. (2001). Hidrovía: contaminación e impacto ambiental Documento digital: http://www.unne.edu.ar/unnevieja/Web/cyt/cyt/2001/1-Sociales/S-019.pdf

Perea Borda, J. (1998). Los ríos nos unen: Integración Fluvial Suramericana. Bogotá, Colombia: CAF. Recuperado de: http://scioteca.caf.com/handle/123456789/868

Pinto Cornejo, P. (noviembre 2018). Complementación económica y mercados del Corredor Bioceánico Central Coquimbo-Porto Alegre. Seminario Internacional -Región de Coquimbo: Plataforma de Inversión y Puente del CBC en el Asia Pacífico, La Serena, Chile. Recuperado de: http://www.eciem.cl/assets/sitio/doc upload/TPanel/Pablo\%20Pinto.pdf

Rofman, A. \& Romero, L. (1997). Sistema socioeconómico y estructura regional en la Argentina. Buenos Aires, Argentina: Amorrortu.

Saguier, E. (2015). Fragmentación del espacio Amazónico y criminal negligencia de nuestros dirigentes en la caracterización de las hidrovías de América Latina. Salta 21 Cultura y Actualidad. Recuperado de: http://salta21.com/Fragmentacion-delespacio.html

Santos, M. (1979). O espaço dividido: os dois circuitos da economia urbana dos países subdesenvolvidos. Río de Janeiro, Brasil: Francisco Alves.

Santos, M. (1993). Los espacios de la globalización. Anales de Geografía de la Universidad Complutense, (13), 69-77. Recuperado de: https://revistas.ucm.es/index.php/AGUC/article/viewFile/AGUC9393110069A/31671

Santos, M. (1996). De la totalidad al lugar. Barcelona, España: Oikos-Tau.

Santos, M. (2004). Por otra globalización: del pensamiento único a la conciencia universal. Bogotá, Colombia: Convenio Andrés Bello.

Silveira, M. L. (2011). Territorio y ciudadanía: reflexiones en tiempo de globalización. UNI-PLURINERSIDAD, 11(3). Recuperado de: https://aprendeenlinea.udea.edu.co/revistas/index.php/unip/article/view/11833/10748

Silveira, M. L. (2016). Constitución de los circuitos de la economía urbana en la globalización. Revista Universitaria de Geografía, 25(2), 79-102. Recuperado de: http://bibliotecadigital.uns.edu.ar/pdf/reuge/v25n2/v25n2a04.pdf

Stancich, E. (2005). Los dueños del río. La hidrovía Paraguay-Paraná: el negocio de los recursos en América Latina. Ecología Política, (31), 27-39. Recuperado de: https://www.ecologiapolitica.info/novaweb2/wpcontent/uploads/2016/08/031 TallerEcol ogista 2006.pdf 
Stancich, E. (2007). Los desastres que vienen por la Hidrovía Paraguay-Paraná . Revista Biodiversidad, 52, 28-30 Recuperado de: https://www.grain.org/es/article/entries/1182-los-desastres-que-vienen-por-la-hidroviaparaguay-parana

Terrazas, R. (2016). Hidrovías para el desarrollo y la integración suramericana. Bogotá Colombia: CAF. Recuperado de: http://scioteca.caf.com/bitstream/handle/123456789/919/CAFHidrovias.pdf?sequence= 1\&isAllowed $=y$

Wilmsmeier, G. (2015). Geografía del transporte de carga. Evolución y desafíos en un contexto global cambiante. CEPAL. Recuperado de: http://repositorio.cepal.org/bitstream/handle/11362/39660/S1501002 es.pdf;jsessionid =92257797C53E30A59964524B9BE78830? sequence $=1$

Zibechi, R. (2012). La nueva geopolítica del capital documento digital. América Latina en movimiento. Recuperado de: https://www.alainet.org/es/active/54196

Zuberman, F. (septiembre 2012). La Cuenca del río de la Plata como núcleo central de la producción mundial de soja. $\mathrm{V}$ Congreso Iberoamericano sobre desarrollo y ambiente de Redibec / V jornadas de la Asociación Argentino Uruguaya de Economía Ecológica, Santa Fe, Argentina Recuperado de: https://www.researchgate.net/publication/324040928 La cuenca del Rio Parana co mo nucleo central de la produccion mundial de soja

Zugaib, E. (2006). A Hidrovía Paraguai - Paraná e seu significado para a diplomacia Sul-Americana do Brasil (Tesis de posgrado), Instituto Rio Branco. Recuperado de: http://www.dominiopublico.gov.br/download/texto/al000188.pdf

Álvarez, Álvaro. Doctor en Geografía por la Facultad de Humanidades y Ciencias de la Educación de la UNLP. Licenciado en Relaciones Internacionales y Magíster en Cs. Sociales por la Facultad de Ciencias Humanas de la UNCPBA. Becario Pos Doctoral del CONICET, miembro del Centro de Investigaciones Geográficas (FCH- UNCPBA), y miembro del Instituto de Geografía Historia y Ciencias Sociales (CONICET- UNCPBA). Docente en la Cátedra Geografía de América Latina (FCH - UNCPBA). Correo electrónico: alvaroa22@yahoo.com.ar 